\title{
A criterion for oscillations in the solutions of the polytropic Lane-Emden equations
}

\author{
Dimitris M Christodoulou', Qutaibeh D Katatbeh ${ }^{2 *}$ and James Graham-Eagle
}

*Correspondence:
qutaibeh@just.edu.jo
2Department of Mathematics and
Statistics, Jordan University of
Science and Technology, Irbid,
22110, Jordan
Full list of author information is
available at the end of the article

available at the end of the article

\begin{abstract}
We have previously formulated a simple criterion for deducing the intervals of oscillations in the solutions of second-order linear homogeneous differential equations. In this work, we extend analytically the same criterion to superlinear Lane-Emden equations with integer polytropic indices $n>1$. We confirm the validity of the analytical results by solving numerically both the cylindrical and the spherical Lane-Emden equations subject to the usual astrophysical boundary conditions for self-gravitating fluids.
\end{abstract}

MSC: $34 \mathrm{~A} 25 ; 34 \mathrm{~A} 34 ; 76 \mathrm{~N} 15 ; 85 \mathrm{~A} 30$

Keywords: oscillations; nonlinear differential equations; analytical theory; transformations; Lane-Emden differential equations

\section{Introduction}

The ordinary second-order linear homogeneous differential equations of mathematical physics

$$
y^{\prime \prime}+b(x) y^{\prime}+c(x) y=0
$$

can all be transformed to the canonical form

$$
u^{\prime \prime}+q(x) u=0
$$

where the primes denote derivatives with respect to the independent variable $x$,

$$
q=-\frac{1}{4}\left(b^{2}+2 b^{\prime}-4 c\right)
$$

and $y(x)=u(x) \exp \left(-\frac{1}{2} \int b(x) d x\right)$ ([1]; [2], Section 10.2; [3], Section XI.1). The canonical form (2) condenses the coefficients of equation (1) into $q(x)$ and 'oscillation theory' focuses on this coefficient in order to derive the oscillatory properties of the solutions of equation (1) (see the reviews in $[4,5]$ and references therein).

In recent work [6, 7], we showed that the canonical form is degenerate in the sense that different equations of the form (1) can be transformed to the same canonical form. This is evident from equation (3) in which $q(x)$ is the result of combining two unrelated functions

(c) 2016 Christodoulou et al. This article is distributed under the terms of the Creative Commons Attribution 4.0 International License (http://creativecommons.org/licenses/by/4.0/), which permits unrestricted use, distribution, and reproduction in any medium, provided you give appropriate credit to the original author(s) and the source, provide a link to the Creative Commons license, and indicate if changes were made. 
$b(x)$ and $c(x)$. Furthermore, the derivative $b^{\prime}(x)$ in equation (3) sometimes acts as damping (when $b^{\prime}>0$ ) and other times enhances oscillations in the solutions (when $b^{\prime}<0$ ). We worked around these ambiguities by transforming equation (2) to a form with constant damping (equation (1) with $b=$ constant), and then we transformed again to a new canonical form in which the constant term $b$ acted unambiguously as damping opposing oscillatory tendencies in the solutions, just as it does in the well-understood case of the damped harmonic oscillator (equation (1) with $b, c=$ constant). This procedure was very successful in deducing the precise intervals of oscillations in the solutions of the general form (1).

In the last step of the procedure, a generalized Euler transformation of the independent variable $x$ was used $[6,7]$ :

$$
x=c_{1}+c_{2} \exp (k t)
$$

where $c_{1}, c_{2}$, and $k$ are arbitrary constants, and a criterion for the intervals of oscillations in the solutions was established:

$$
q(x)>\frac{1}{4\left(x-c_{1}\right)^{2}} .
$$

Only the constant $c_{1}$ appears in the criterion and corresponds to a 'horizontal shift' of the independent variable $x$ in equation (4). For equations with singularities at the origin, $c_{1}$ can be set to zero and then the criterion (5) reduces to the simple form

$$
q(x)>\frac{1}{4 x^{2}}
$$

In this case, we can also choose $c_{2}=1$ and $k=1$ in equation (4) and then the change of the independent variable $x$ takes the form of the classical Euler transformation

$$
x=\exp (t)
$$

for which the investigation of the interval $t \in(-\infty,+\infty)$ in the transformed equation corresponds to searching for oscillatory solutions in the interval $x \in(0,+\infty)$ of the original equation (1).

In this work, we extend the applicability of the criterion (6) to nonlinear equations of the Lane-Emden type [8-11]

$$
y^{\prime \prime}+\frac{D-1}{x} y^{\prime}+y^{n}=0 \quad(x \geq 0)
$$

where $n \neq 0,1$ is the polytropic index and $D$ is the dimension of space ( $D=2$ for a cylindrical radius $x$ and $D=3$ for a spherical radius $x$ ). The properties of the solutions of this equation have been studied intensely by many researchers in recent years (see [12-17] and references therein) because analytic solutions are hard to come by (see [11]). In the cited works, a wide variety of complicated mathematical techniques have been employed, as well as direct numerical integrations of the Cauchy problem have been carried out to tackle its many applications. 
In equation (8), the coefficient of $y^{\prime}$ is $1 / x$ in the cylindrical case and $2 / x$ in the spherical case. These coefficients were discussed in detail in [6] for the simpler case of linear equations: the cylindrical inertial term $y^{\prime} / x$ represents the complete absence of damping, while the spherical inertial term $2 y^{\prime} / x$ results in the elimination of the $b$-dependent terms from equation (3) so that $q(x) \equiv c(x)$. In the linear case, the two inertial terms lead to dramatically different criteria for oscillatory solutions ( $c>0$ and $c>1 /\left(4 x^{2}\right)$, respectively).

It is not obvious that the results and the conclusions of the linear case carry over to nonlinear cases as well, but this turns out to be the case for the real solutions of the LaneEmden equations (8) with integer values of $n>1$. In Section 2, we derive analytically the criteria for oscillations in the solutions of the polytropic equation (8) and we test their validity by performing numerical integrations for integer values of $n>1$. Then, in Section 3, we discuss these polytropic results and their striking difference from the isothermal LaneEmden case [8-11] and the negative integer $n$ case, for which we know that all solutions are strictly nonoscillatory. Finally, in Section 4, we summarize our conclusions.

\section{Criteria for polytropic Lane-Emden equations}

We carry out the procedure described in [6] to equation (8): First we transform this equation to a 'canonical form' by eliminating the first-derivative term. We obtain the form of equation (2) in which the coefficient $q(x)$ is given by

$$
q(x)=y^{n-1}-\frac{1}{4 x^{2}}(D-1)(D-3),
$$

and $u(x)=y(x) x^{(D-1) / 2}$. We observe that the nonlinear term of the Lane-Emden equations has introduced $y^{n-1}(x)$ into $q(x)$.

Next we choose $c_{1}=0$ and $c_{2}=k=1$ in equation (4), in which case the transformation of $x$ is given by equation (7) and the criterion for oscillatory solutions over the interval $x \in(0,+\infty)$ is given by equation (6).

Finally, we substitute equation (9) into equation (6) and the criterion for oscillatory solutions becomes

$$
y^{n-1}>\left(\frac{D-2}{2 x}\right)^{2} .
$$

We now assume that $n$ is an integer larger than 1 in which case the solutions of equation (8) are real functions of $x \geq 0$. The linear case $n=1$ has been covered by the analysis in [6] and it can be solved analytically as well $[10,11,18]$. The $n=1$ solutions for $D=$ 2, 3 all oscillate about the $x$-axis irrespective of the adopted boundary conditions. On the other hand, negative integer values of $n$ are excluded for the following reason: In both the linear case (equation (1)) and the Lane-Emden case (equation (8)), when oscillatory solutions occur, their oscillations always develop about a trivial solution $(y=0$ in most well-known cases; see [6]) which serves as a baseline for the oscillations. If a trivial solution does not exist, then all solutions are nonoscillatory. As a result, if $n$ is a negative integer, the solutions of the Lane-Emden equations are strictly monotonic because then the equations do not admit $y=0$ as a trivial solution. For the same reason, the so-called isothermal Lane-Emden equations [8-11] with $D=2,3$ also have strictly nonoscillatory solutions. In these equations, the term $y^{n}$ in equation ( 8$)$ is replaced by $\exp (y) \neq 0$, which prevents the existence of a trivial solution. 


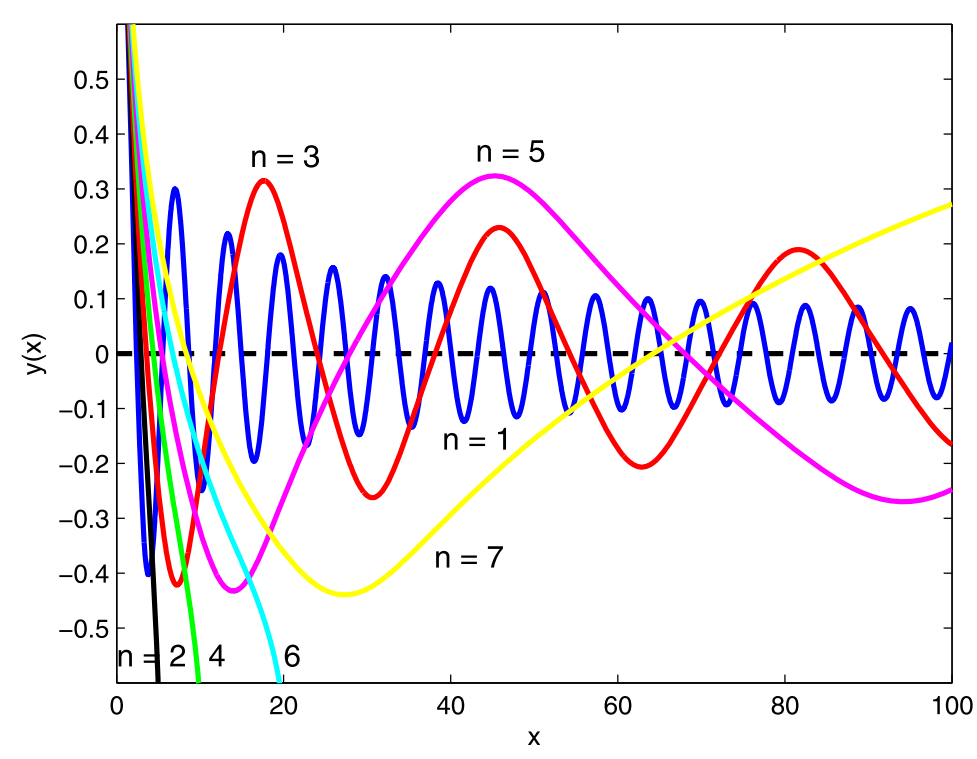

Figure 1 The numerical solutions of the cylindrical equation (8) with $D=2$, integer $n=1-7$, and boundary conditions $y(0)=1$ and $y^{\prime}(0)=0$. The solutions with odd $n$ are oscillatory about $y=0$, while the solutions with even $n$ are monotonic.

\subsection{Cylindrical case}

For $D=2$, equation (10) reduces to

$$
y^{n-1}>0
$$

This criterion predicts oscillatory solutions in all cases with an odd integer $n>1$, while solutions with even values of $n$ must be monotonic since they cross below the $x$-axis, become negative, and fail to satisfy the criterion. Numerical integrations using the usual astrophysical boundary conditions $y(0)=1, y^{\prime}(0)=0[10]$ confirm this prediction (Figure 1).

\subsection{Spherical case}

For $D=3$, equation (10) reduces to

$$
y^{n-1}>\frac{1}{4 x^{2}} .
$$

This criterion is more difficult to decipher. We distinguish two cases:

(a) For even values of $n>1$, equation (12) becomes

$$
y>\left(\frac{1}{2 x}\right)^{\frac{2}{n-1}}
$$

and it implies that the solutions will be nonoscillatory because either they become negative if they cross the $x$-axis (i.e., the trivial solution $y=0$ ) and then the inequality is not satisfied; or they remain positive if they do not cross the $x$-axis, and then they cannot oscillate about $y=0$ (as is the case for even values of $n \geq 6$; see Figure 2). 


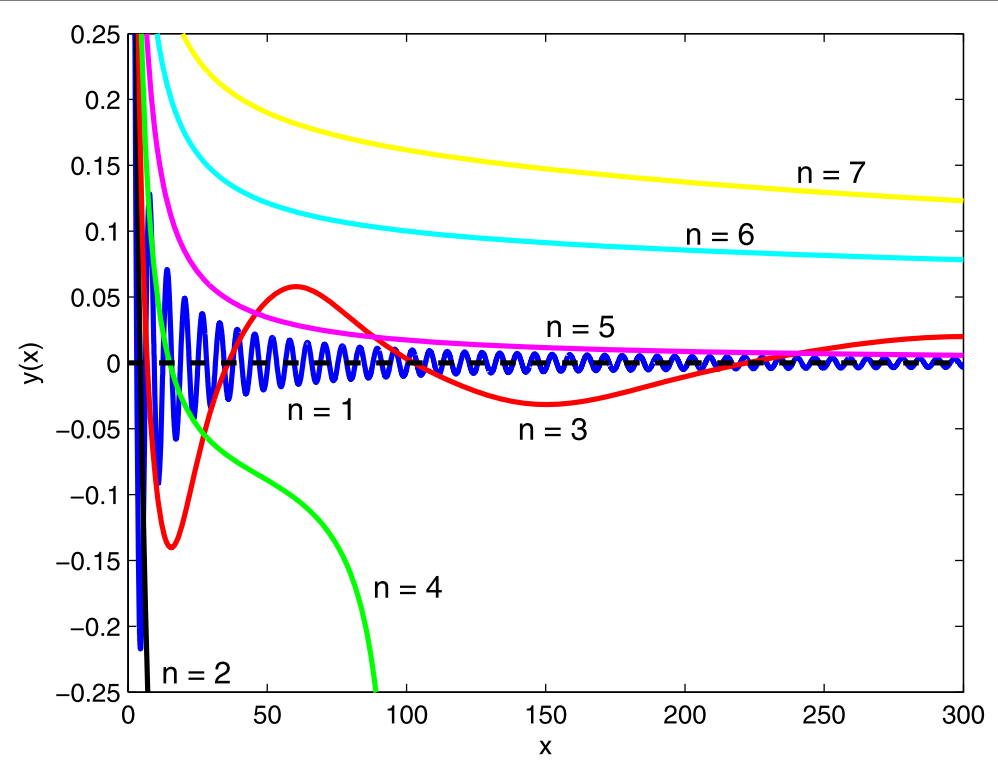

Figure 2 The numerical solutions of the spherical equation (8) with $D=3$, integer $n=1-7$, and boundary conditions $\boldsymbol{y}(\mathbf{0})=\mathbf{1}$ and $\boldsymbol{y}^{\prime}(\mathbf{0})=\mathbf{0}$. For $n=1$ and $n=5$, the solutions are also known analytically [10]. Surprisingly, the solution for $n=3$ is the only nonlinear solution that is oscillatory.

(b) For odd values of $n>1$, equation (12) becomes

$$
|y|>\left(\frac{1}{2 x}\right)^{\frac{2}{n-1}}
$$

and it implies that a solution will be oscillatory only if it crosses repeatedly outside the no-oscillation region defined by the curves $y_{ \pm}= \pm(2 x)^{-2 /(n-1)}$.

Presently, we know enough details as regards the solutions of the spherical Lane-Emden equation to reach the surprising conclusion that only the solutions with $n=3$ may be oscillatory: For all spherical polytropes with $n>3$, there exists a singular solution of equation (8) with $D=3$ ([10], Section IV.3(c); [11], Section 2.3.4) of the form

$$
y=\left[\frac{2(n-3)}{(n-1)^{2} x^{2}}\right]^{\frac{1}{n-1}} \quad(n>3) .
$$

For odd integers $n \geq 5$, the physical solutions of the boundary-value problem $(y(0)=1$, $y^{\prime}(0)=0$ ) remain near this singular solution with increasing $x$ and match it asymptotically with increasing $n$. Substituting equation (15) into the criterion (12), we find that

$$
(n-5)^{2}<0 \quad(n>3)
$$

a condition that is not satisfied for any value of $n$. Therefore, the criterion for oscillatory solutions is not satisfied by the singular solution for any odd value of $n>3$ and this includes the marginal case with $n=5$. Since the solutions of the boundary-value problem for $n \geq 5$ trace this monotonic singular solution and remain close to it, they are expected to be nonoscillatory as well. 


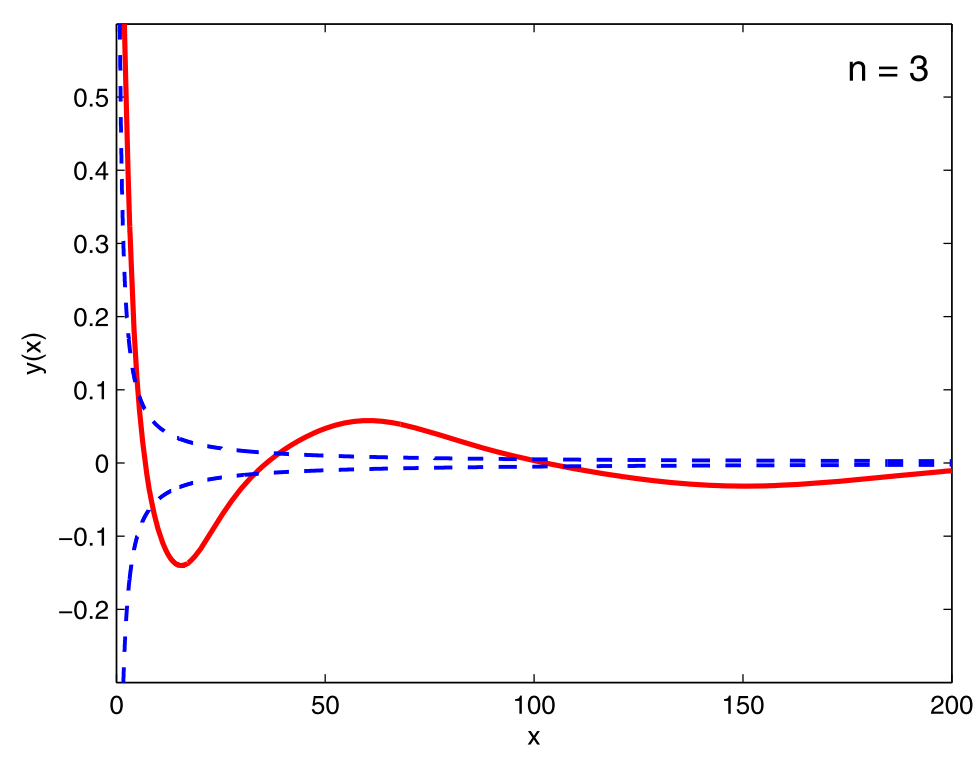

Figure 3 The numerical solution of the spherical equation (8) with $D=3, n=3$, and boundary conditions $y(0)=1$ and $y^{\prime}(0)=0$. The solution turns around every time it overshoots the region of no oscillations between the dashed curves $y_{ \pm}= \pm 1 /(2 x)$.

Numerical integrations using the usual astrophysical boundary conditions $y(0)=1$, $y^{\prime}(0)=0[10]$ confirm the above predictions: Figure 2 shows that the solution for $n=3$ is the only nonlinear solution that is oscillatory. Figure 3 shows this $n=3$ numerical solution (solid line) in relation to the no-oscillation region $|y| \leq 1 /(2 x)$ (between the dashed lines) that was predicted by equation (14). Every time the solution overshoots the no-oscillation region, it turns around thereby producing successive extrema. Similarly, Figure 4 shows the $n=5$ marginal solution (solid line) in relation to the no-oscillation region $|y| \leq 1 / \sqrt{2 x}$ (between the dashed lines) that was predicted by equation (14). As was expected, this solution enters the no-oscillation region, remains in it, and continues to be monotonic for larger values of $x$.

\section{Discussion}

We have presented an analysis of the oscillatory properties of the solutions of the nonlinear polytropic Lane-Emden equations that describe the equilibrium structures of selfgravitating fluid spheres and cylinders [8-18]. The analysis makes use of a procedure that was described in $[6,7]$ for second-order linear homogeneous differential equations. It turns out that the same procedure is also valid for nonlinear homogeneous equations, provided that they possess a trivial solution of the form $y=0$ that serves as a baseline for oscillatory solutions. This requirement is satisfied by the superlinear polytropic LaneEmden equations with indices $n>1$.

We limited our analysis to integer values of $n$ for which the solutions are real functions of the radius $x$. We have shown analytically that the oscillation criteria derived in the linear case [6] carry over to the $n>1$ polytropic cases as well. This is the direct result of the behavior of the inertial terms in a cylindrical $\left(y^{\prime} / x\right)$ and a spherical $\left(2 y^{\prime} / x\right)$ geometry:

(a) In the cylindrical case $(D=2$ in equation (8)), the inertial term indicates that there is no damping of the oscillations, just as in the linear cases of the Bessel differential equation 


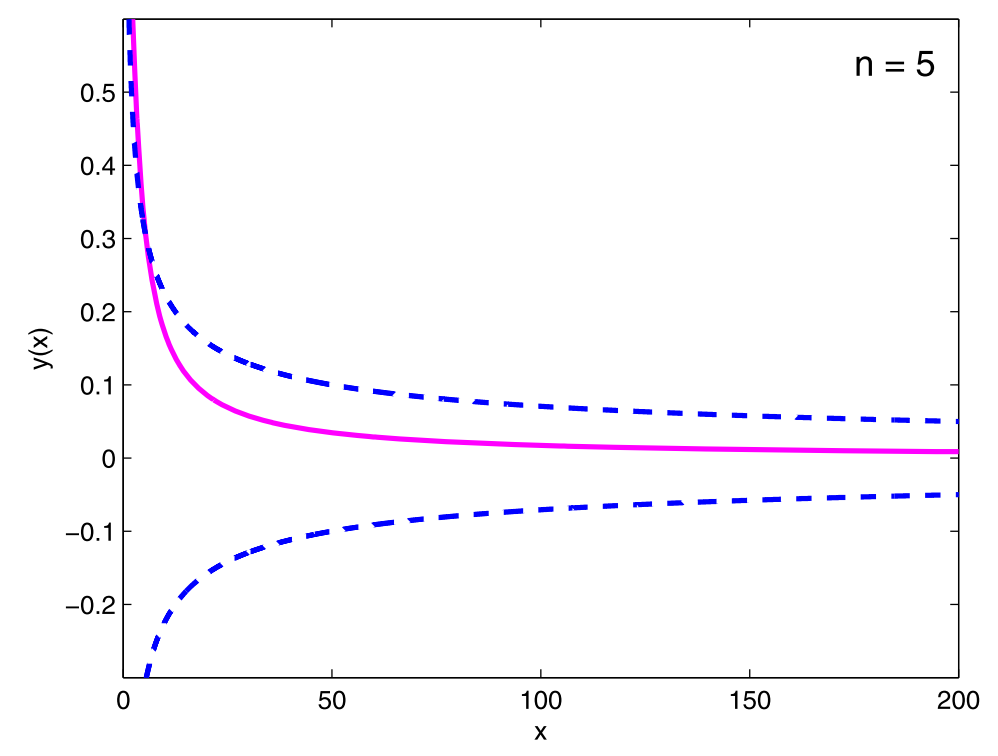

Figure 4 The known analytical solution $y=1 / \sqrt{1+x^{2} / 3}$ [10] of the spherical equation (8) with $D=3$, $n=5$, and boundary conditions $y(0)=1$ and $y^{\prime}(0)=0$. The solution enters and stays in the region between the dashed curves $y_{ \pm}= \pm 1 / \sqrt{2 x}$ where it cannot oscillate. The upper dashed curve $y_{+}$also represents the singular solution (15) with $n=5$.

and the 'fuel cell' equation $[6,19]$. The criterion (6) for oscillatory solutions then reduces to $c(x)>0$, where $c(x)=y^{n-1}(x)$ as indicated by a comparison between equations (8) and (1) with $b(x)=1 / x$. In this case, we have shown that only the solutions with odd integers $n>1$ are oscillatory and the numerical solutions of equation (8) with $D=2$ have confirmed this result (Figure 1).

(b) In the spherical case ( $D=3$ in equation (8)), the inertial term leads to a cancellation of the $b$-dependent terms in the canonical form (equations (2) and (3)). The criterion (6) for oscillatory solutions then reduces to $c(x)>1 /\left(4 x^{2}\right)$, where $c(x)=y^{n-1}(x)$ and $b(x)=2 / x$ in equation (3). In this case, we have obtained a surprising result: the $n=3$ case is the only nonlinear case whose solutions may be oscillatory. The numerical solutions of equation (8) with $D=3$ have confirmed this curious result (Figures 2, 3, and 4).

In the analysis presented in Sections 2.1 and 2.2, we have considered cylindrical and spherical polytropes, respectively, with integer values of the index $n>1$. There was no need to consider negative integer values of $n$ because then equation (8) no longer possesses a trivial solution of the form $y=0$. As we discussed above, the absence of a trivial solution implies that all solutions will be strictly monotonic. For the solutions of the Lane-Emden equations (8) with integer values of $n \leq-1$, we have verified such a monotonic behavior by numerical integrations of several cases (not shown here).

The isothermal Lane-Emden equations [8-11] also have strictly nonoscillatory solutions because they do not admit a trivial solution. These equations are characterized by an exponential nonlinearity of the form $\exp (y)$ (instead of $y^{n}$ in equation (8)) that precludes the $y=0$ solution which is necessary (but not sufficient) for the appearance of oscillatory solutions. Taking all this together, the above results lead to another surprising conclusion: the presence or the absence of trivial solutions in differential equations of the second order is an important qualifier of the properties of the solutions of the physical Cauchy problem; thus, they should not be ignored, as their name signifies. Lately, we have come to call them 
intrinsic solutions because when the differential equations admit such solutions, they do so with no regard to any boundary or initial conditions that may be imposed externally by the Cauchy problem.

\section{Conclusions}

We have derived criteria for oscillations in the solutions of the nonlinear polytropic LaneEmden equations (8) [8-18] in a cylindrical and a spherical geometry (equations (11) and (12), respectively). These inequalities show that in the cylindrical case, oscillatory solutions occur for all odd polytropic indices $n>1$ (Section 2.1, Figure 1), while in the spherical case, only the $n=3$ polytrope may have oscillatory solutions (Section 2.2, Figure 2). In both cases, the oscillations develop about the trivial solution $y=0$ of the differential equation and the existence of such an intrinsic solution is a necessary condition for the appearance of oscillations. The polytropic equations with negative integer values of $n$ and the isothermal Lane-Emden equations [8-11] do not admit a trivial solution, therefore their solutions are all nonoscillatory.

\section{Competing interests}

The authors declare that they have no competing interests.

\section{Authors' contributions}

All authors drafted the manuscript, and they read and approved the submitted version.

\section{Author details}

${ }^{1}$ Department of Mathematical Sciences, University of Massachusetts Lowell, Lowell, MA 01854, USA. ${ }^{2}$ Department of Mathematics and Statistics, Jordan University of Science and Technology, Irbid, 22110, Jordan.

\section{Acknowledgements}

During this research project, DMC and JG-E were supported by the University of Massachusetts Lowell whereas QDK was on a sabbatical visit and was fully supported by the Jordan University of Science and Technology. The authors would like to thank the referees for their constructive criticisms, which led to improvements of this paper.

Received: 16 February 2016 Accepted: 13 May 2016 Published online: 04 June 2016

\section{References}

1. Abramowitz, M, Stegun, IA (eds.): Handbook of Mathematical Functions with Formulas, Graphs, and Mathematical Tables. Dover, New York (1972)

2. Whittaker, ET, Watson, GN: A Course of Modern Analysis, 3rd edn. Cambridge University Press, Cambridge (1920)

3. Hartman, P: Ordinary Differential Equations. Wiley, New York (1964)

4. Agarwal, RP, Grace, SR, O'Regan, D: Oscillation Theory for Second Order Linear, Half-Linear, Superlinear and Sublinear Dynamic Equations. Kluwer Academic, Dordrecht (2002)

5. Wong, JSW: On second-order nonlinear oscillation. Funkc. Ekvacioj 11, 207-234 (1968)

6. Christodoulou, DM, Graham-Eagle, J, Katatbeh, QD: A program for predicting the intervals of oscillations in the solutions of ordinary second-order linear homogeneous differential equations. Adv. Differ. Equ. (2016). doi:10.1186/s13662-016-0774-x

7. Katatbeh, QD, Christodoulou, DM, Graham-Eagle, J: The intervals of oscillations in the solutions of the radial Schrödinger differential equation. Adv. Differ. Equ. (2016). doi:10.1186/s13662-016-0777-7

8. Lane, JH: On the theoretical temperature of the Sun. Am. J. Sci. Arts 50, 57-74 (1870)

9. Emden, R: Gaskugeln. Teubner, Leipzig (1907)

10. Chandrasekhar, S: An Introduction to the Study of Stellar Structure. University of Chicago Press, Chicago (1939)

11. Horedt, GP: Polytropes. Kluwer Academic, Dordrecht (2004)

12. Benguria, RD: The Lane-Emden equation revisited. Contemp. Math. 327, 11-19 (2003)

13. Farina, A: On the classification of solutions of the Lane-Emden equation on unbounded domains of $\mathbb{R}^{N}$. J. Math. Pures Appl. 87, 537-561 (2007)

14. Van Gorder, RA, Vajravelu, K: Analytic and numerical solutions to the Lane-Emden equation. Phys. Lett. A 372 , 6060-6065 (2008)

15. Parand, K, Dehghan, M, Rezaei, AR, Ghaderi, SM: An approximation algorithm for the solution of the nonlinear Lane-Emden type equations arising in astrophysics using Hermite functions collocation method. Comput. Phys. Commun. 181, 1096-1108 (2010)

16. Parand, K, Rezaei, AR, Taghavi, A: Lagrangian method for solving Lane-Emden type equation arising in astrophysics on semi-infinite domains. Acta Astronaut. 67, 673-680 (2010)

17. Kumar, N, Pandey, RK, Cattani, C: Solution of the Lane-Emden equation using the Bernstein operational matrix of integration. Int. Sch. Res. Not. Astron. Astrophys. 2011, Article ID 351747 (2011) 
18. Robe, H: Équilibre et oscillations des cylindres polytropiques compressibles en rotation. Ann. Astrophys. 31, 549-558 (1968)

19. Bailey, PB, Billingham, J, Cooper, RJ, Everitt, WN, King, AC, Kong, Q, Wu, H, Zettl, A: On some eigenvalue problems in fuel-cell dynamics. Proc. R. Soc. Lond. A 459, 241-261 (2003)

Submit your manuscript to a SpringerOpen ${ }^{\circ}$ journal and benefit from:

- Convenient online submission

Rigorous peer review

- Immediate publication on acceptance

- Open access: articles freely available online

- High visibility within the field

- Retaining the copyright to your article

Submit your next manuscript at $\boldsymbol{s p r i n g e r o p e n . c o m ~}$ 